\title{
Water chemistry on surface defect sites: Chemidissociation versus physisorption on $\mathrm{MgO}(001)$
}

\author{
C. A. Scamehorn \\ MS Kl-90, Molecular Sciences Research Center, Pacific Northwest Laboratory, Richland, \\ Washington 99352 \\ N. M. Harrison \\ Daresbury Laboratory, Daresbury, Warrington WA4 4AD, United Kingdom \\ M. I. McCarthy \\ MS K1-90, Molecular Sciences Research Center, Pacific Northwest Laboratory, Richland, \\ Washington 99352
}

(Received 14 February 1994; accepted 30 March 1994)

The following paper presents the results of a theoretical study that probed the chemistry of water at structural defects on the MgO (001) surface. The computational technique used was periodic Hartree-Fock (PHF) theory with density functional based correlation corrections. The adsorption energies for water adsorbed on isolated corner, edge, and surface sites on the $\mathrm{MgO}$ surface were compared to the hydroxylation energies for the same sites. As stated in a previous paper, the binding of water to the perfect surface is exothermic by $4.1-5.6 \mathrm{kcal} / \mathrm{mol}$ whereas hydroxylating the perfect surface was endothermic by $24.5 \mathrm{kcal} / \mathrm{mol}$. At step-edge sites, the process of water adsorption is exothermic and comparable in magnitude to the hydroxylation of these sites. The binding energies associated with water bound to the step-edge are $6.5-10.5 \mathrm{kcal} / \mathrm{mol}$, and hydroxylation of this site is exothermic by $7.3 \mathrm{kcal} / \mathrm{mol}$. At corner sites we find a strong preference for hydroxylation. The binding of water to a corner is exothermic by $20.7 \mathrm{kcal} / \mathrm{mol}$, and hydroxylation is exothermic by $67.3 \mathrm{kcal} / \mathrm{mol}$. Mulliken populations indicate that the formation of a hydroxylated surface is governed by the stability of the hydroxyl bond where a hydrogen is bonded to a surface oxygen ion. As the coordination number of this oxygen binding site decreases, its ionic character also decreases, and it forms a more stable bond with the incoming hydrogen. This trend is confirmed by the densities of states for these sites. Finally, hydroxylation of the perfect (001) surface was examined as a function of lattice dilation. It was determined that, as the lattice constant increases, hydroxylation becomes more energetically favorable. This may be important in interpreting experimental thin-film results where the lattice constant of the substrate upon which the $\mathrm{MgO}$ film is deposited is slightly larger than that of bulk $\mathrm{MgO}$.

\section{INTRODUCTION}

Structural defects are known to play an important role in determining the surface chemistry of oxides. The advent of experimental thin-film techniques has advanced this knowledge by permitting the use of electron scattering techniques to characterize the properties of oxide surfaces by reducing the surface charging problems traditionally associated with these methods. Although thin films have been shown to qualitatively mimic the surfaces of bulk materials, they may display quantitatively different surface properties from clean (cleaved or grown) crystals. A full characterization of the properties of oxide films is part of ongoing experimental efforts. The present work attempts to resolve some of these issues by examining (theoretically) the reactivity of different surface defect sites that may be present in varying concentrations in thin $\mathrm{MgO}$ films and cleaved $\mathrm{MgO}(001)$ crystals. The aim of this study is to account for conflicting experimental results on the chemidissociation of water on $\mathrm{MgO}$ surfaces and to obtain a general understanding of the role structural properties play in interfacial chemistry.

For the case of water adsorbed on the MgO (001) surface, IR (infrared) studies of water on $\mathrm{MgO}$ smoke $^{1}$ and ultraviolet photoemission spectroscopy (UPS) and x-ray pho- toemission spectroscopy (XPS) studies of water on a singlecrystal surface ${ }^{2}$ have shown no evidence of hydroxylation of this surface. In contrast, a high-resolution electron energy loss spectroscopic) (HREELS) study on an ultrathin magnesium oxide film ${ }^{3}$ and an IR study of water on $\mathrm{MgO}$ powders ${ }^{4}$ indicates that hydroxylation does indeed occur. Precise characterization of surface structure is still a complicated experimental task making it difficult to compare results obtained from surfaces prepared using different methods. We have undertaken a set of theoretical studies to determine the energetics associated with the hydroxylation of the $\mathrm{MgO}(001)$ surface and to characterize the chemistry at several structural defects that may be probed by the different experiments. In a previous paper, referred to henceforth as $\mathrm{Pl}$, we presented similar results for the perfect $\mathrm{MgO}(001)$ surface. ${ }^{5}$ In this paper, we present a description of possible structural defects and the chemistry of water bound at these sites. The three defects studied were step-edges, step-corners, and dilated lattices.

\section{COMPUTATIONAL DETAILS}

The calculations presented here were carried out using the program CRYSTAL92, ${ }^{6}$ which is based on a method de- 
signed to describe crystalline solids at an $a b$ initio level of theory by solving the Hartree-Fock-Roothaan equations subject to periodic boundary conditions. This method has also been extended to include a posteriori density functional correlation corrections to the ground-state energies. ${ }^{7,8}$ Correlation corrected periodic Hartree-Fock theory has been used to study the chemistry of $\mathrm{Cl}_{2}^{9}$ and $\mathrm{H}_{2} \mathrm{O}^{5}$ on the perfect $(001)$ $\mathrm{MgO}$ surface.

In our previous work (P1), we examined the defect free (001) MgO surface, and many aspects of the model used in that study were retained in the current study of defect sites on the surface. The linear combination of atomic orbitals (LCAO) basis sets used to describe all of the surfaces are expressed as 8-51 $\mathrm{G}$ for magnesium and 8-61 $\mathrm{G}$ for oxygen. ${ }^{10}$ These basis sets have been specially developed for use with crystalline systems and have been successfully used in previous studies on $\mathrm{MgO}$ surfaces. ${ }^{5,9,11,12}$ The standard Pople $6-31 \mathrm{G}^{*}$ basis set ${ }^{13}$ was used to describe adsorbed and dissociated water on the surface. The water geometry used was optimized for this basis using GAUSSIAN90. ${ }^{14}$

In the current work we model the water chemistry at two different types of surface defect sites, one in which the adsorption sites are fourfold coordinated (edge sites) and one in which they are threefold coordinated (corner sites). In the case of the fourfold coordinated site, a step one monolayer in thickness is formed along the edge of a surface that is three layers thick, five ions wide, and extends infinitely in one dimension. The threefold coordinated corner sites are constructed by adding a square consisting of two $\mathrm{MgO}$ formula units (four ions total) to the perfect, three layer (001) surface. The distance between the threefold coordinated defect sites measured from the centers of each defect was varied from 5.95 to $11.89 \AA$ in order to determine the effect of defect density on the surface water chemistry. The positions of the ions comprising the surface defects were optimized at the upper limit of defect concentration using CRYSTAL92, and it was determined that relaxation at these sites is less than $0.5 \%$. Therefore, the lattice spacing of bulk $\mathrm{MgO}$ was used in the calculation involving defect sites to maximize the symmetry of the system. Schematic diagrams of these structures are given in Fig. 1.

A final note on terminology. For the remainder of the paper, the term "perfect surface" refers to the defect-free (001) surface, and the term "clean surface" corresponds to any of the MgO surfaces described here in the absence of water molecules or hydroxyl groups.

\section{RESULTS AND DISCUSSION}

\section{A. Surface defect construction}

In constructing defected magnesium oxide surfaces, we would like to probe configurations that most closely resemble the experimentally prepared surfaces. There is evidence in the literature that even those surfaces prepared by cleavage of a single crystal along the (001) plane contain steps. X-ray studies ${ }^{15}$ have shown that these steps consist of plateaus $\sim 8000 \AA^{2}$ in the case of two layer high steps and $\sim 30000 \AA^{2}$ when the steps are three monolayers high. The presence of steps one atom high was not discussed because

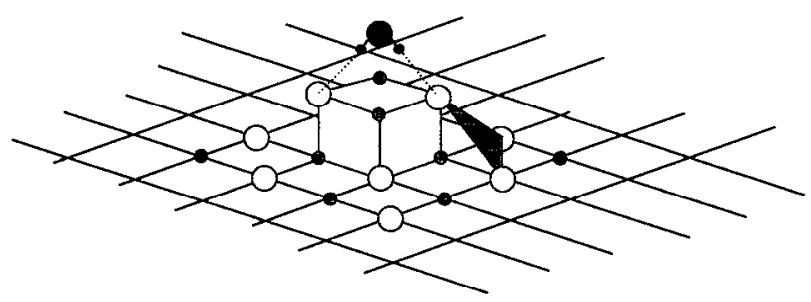

(a)

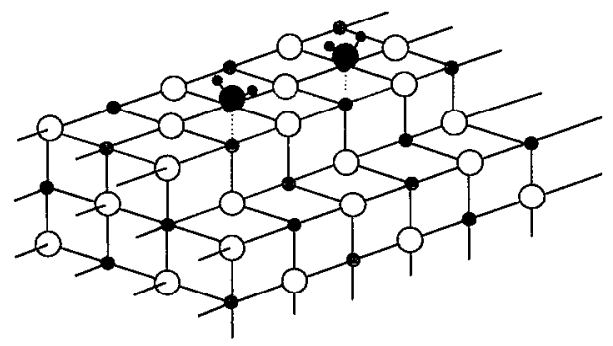

(b)

FIG. 1. Schematic diagrams of the corner (a) and edge (b) defect structures used in this study and the orientations of water molecules over these defects. The shaded triangle in (a) connects three nearest-neighbor oxygen ions ( $d$ $=2.97 \AA$ ).

of the difficulties involved in interpreting very low angle Bragg reflection data. At this point it is not practical to perform $a b$ initio calculations on unit cells containing steps of this magnitude. Our approach to understanding defect chemistry is to study edge and corner sites separately and try to correlate this information with the experimental data. For the calculations involving corner sites, four-ion clusters were distributed on the surface in several concentrations and orientations in order to minimize defect-defect interactions.

To get an estimate of the size of cell needed to minimize the electrostatic interactions between defects, a series of calculations were performed on two periodic arrangements of four-ion units in the absence of the underlying $\mathrm{MgO}$ slab. One set of calculations was done on a set of defects oriented in a corner-to-corner fashion and in the other configurations the defects were arranged in a side-to-side manner (see Fig. 2). For each series of calculations performed, the defectdefect distance was varied over a range of values from 6 to $15 \AA$. The interaction energy between defects was calculated by subtracting the energy of an isolated square of four ions from that of the periodic array of four-ion defects. These energies are plotted as a function of defect-defect distance in Fig. 2, where the distance between defects is defined in both cases as the distance between the center of each four-ion unit and that of its nearest neighbor. Note that in the case where the defects are aligned corner-to-corner the interaction is repulsive, while in the other case where the defects are aligned side-to-side this interaction is attractive. In this figure, the points corresponding to the lattice constants of possible $\mathrm{MgO}$ unit cells are circled. Schematic diagrams of the defected $\mathrm{MgO}$ surface unit cells are given in Fig. 3. 


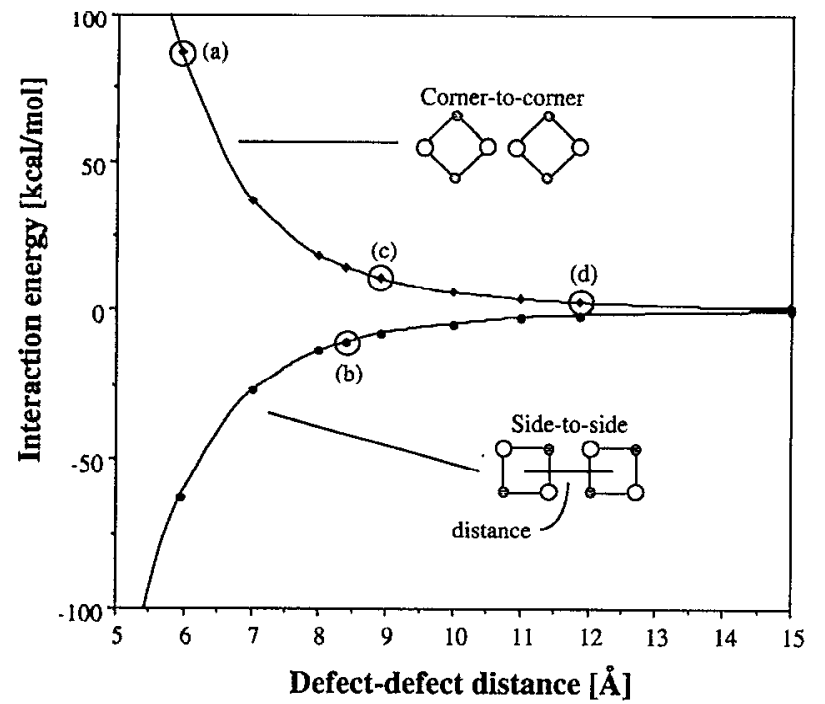

FIG. 2. Defect-defect interaction energies as a function of both distance and orientation. The defects here are periodic arrays of four-ion defects without the supporting $\mathrm{MgO}$ slab beneath them. The circled points indicate the defect-defect spacing corresponding to the unit cells shown in Fig. 3.

Formation energies, $E^{\text {def }}$, for each of the cells were calculated according to

$$
E^{\mathrm{def}}=\frac{E^{\text {tot }}-\left[n_{d}\left(E^{\text {bulk }}\right)+n_{s}\left(E^{\text {slab }}\right)\right]}{2},
$$

where $E^{\text {tot }}$ is the total energy of the unit cell, $n_{d}$ and $n_{s}$ are the number of atoms found in the defect and in the slab, respectively, and $E^{\text {bulk }}$ and $E^{\text {slab }}$ are the energies of bulk $\mathrm{MgO}$ and the $\mathrm{MgO}$ slab normalized to a per atom quantity. A

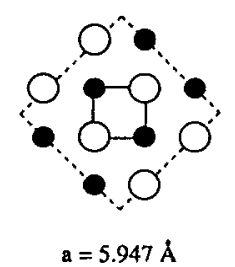

(a)

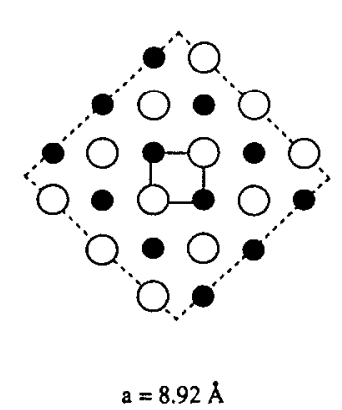

(c)

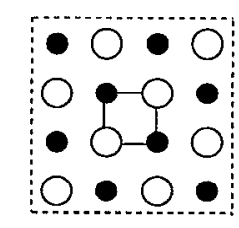

$a=8.41 \AA$

(b)

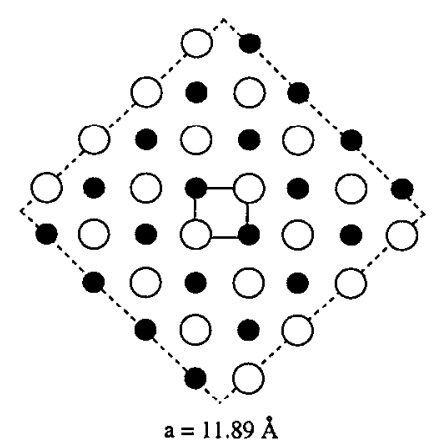

(d)
FIG. 3. Unit cells used for corner defect site calculations.

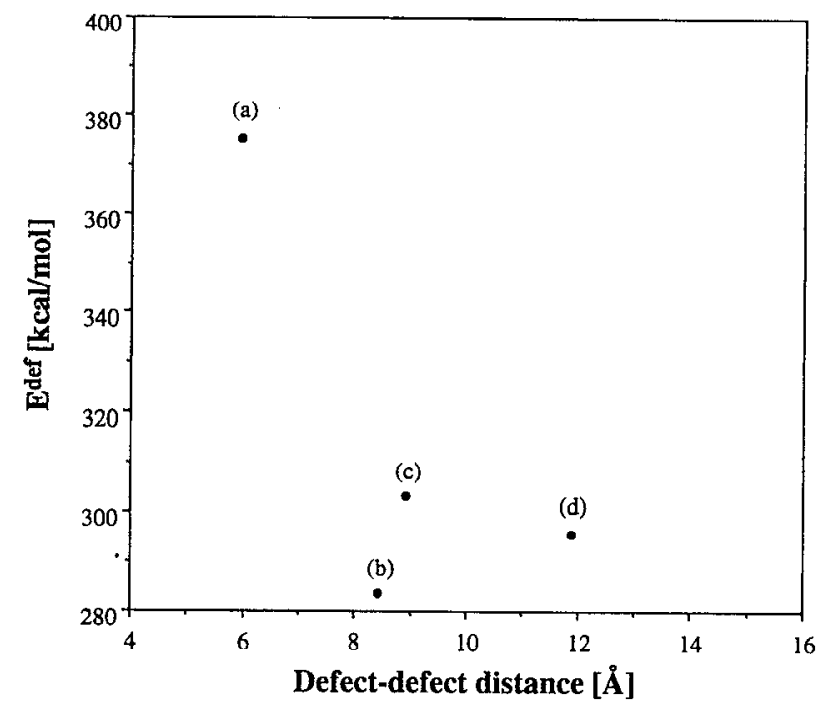

FIG. 4. Defect formation energies in $\mathrm{kcal} / \mathrm{mol}$. This quantity is defined by Eq. (1) in the text. (a), (b), (c), and (d) correspond to the unit cells shown in Fig. 3.

plot of the distance dependence of the formation energies is shown in Fig. 4. Division by a factor of 2 is necessary because the model used consists of a finite thickness slab with two surfaces.

One goal of the calculations performed in this section was to determine which of the four possible $\mathrm{MgO}$ unit cells given in Fig. 3 is the best approximation of an isolated defect. Cell (a) was shown to have a high degree of interaction between defects and will not be studied further. Cell (d) would be the best choice since the defects are farthest apart; however, our available resources restrict us to a smaller cell. The defect-defect interactions for cells (b) and (c) are comparable in magnitude but opposite in sign, one being repulsive and the other being attractive, making a choice between them difficult. Because there is no clear reason to prefer one of these two cells over the other, the surface-water binding energies and hydroxylation energies for both of these surfaces will be examined in the next section.

\section{B. Molecular adsorption versus chemidissociation}

In our study of the perfect magnesium oxide surface (P1), we examined nine orientations of water bound over the surface and found that three of them showed binding of water molecules to that surface. In two of these configurations, the water molecules were oriented with oxygen down over a magnesium ion and these gave Hartree-Fock (HF) binding energies of $4.1-4.5 \mathrm{kcal} / \mathrm{mol}(8.2-11.1 \mathrm{kcal} / \mathrm{mol}$ with the inclusion of a posteriori electron correlation corrections). The other orientation had the water molecule positioned with hydrogens pointing down toward surface oxygens and the water oxygen centered over an interstitial site. This configuration was the most strongly bound with an HF binding energy of $5.6 \mathrm{kcal} / \mathrm{mol}(11.0-12.5$ with correlation corrections). In addition to the calculations studying adsorbed molecular water, we also examined hydroxylation of the per- 
fect surface. Hydroxylated $\mathrm{MgO}$ was found to be energetically unfavorable with respect to the clean surface and isolated water molecule by $24.5 \mathrm{kcal} / \mathrm{mol}$.

The present work examines configurations of water adsorbed at the defect sites which correspond to those that showed significant binding to the perfect surface in P1. In the case of an edge defect, water is adsorbed perpendicular to the surface with oxygen down over a four-coordinated magnesium ion. Binding energies were obtained for one orientation in which the plane of the molecule is perpendicular to the step-edge and for another in which the plane of the molecule is positioned parallel to the step-edge [see Fig. 1(b)]. For binding to corner sites, the water molecule is centered diagonally across the four-ion defect with hydrogen atoms pointing down toward the three-coordinated oxygens. This orientation is analogous to the water geometry found to give the strongest binding to the defect-free $\mathrm{MgO}$ surface in our previous work. All of the defect-water configurations are shown schematically in Fig. 1.

The hydroxylated surfaces were created by positioning a hydroxyl group over each magnesium adsorption site and a hydrogen atom over each oxygen adsorption site. These groups were placed normal to the surface sites. The $\mathrm{O}-\mathrm{H}$ and $\mathrm{Mg}-\mathrm{O}$ bond lengths were optimized in each case but because structural optimization is computationally expensive, the symmetry of the slab was preserved in these calculations forcing the $\mathrm{OH}$ groups to be perpendicular to the surface.

In order to predict whether hydroxylation is thermodynamically favorable at defect sites on the $\mathrm{MgO}(001)$ surface, the energy arising from the adsorption of water at the defect sites is compared to that for the hydroxylation of the same defects. Both adsorption and hydroxylation energies are defined by the following formula:

$$
E=E(\mathrm{MgO})+E\left(\mathrm{H}_{2} \mathrm{O}\right)-E\left(\mathrm{MgO} / \mathrm{H}_{2} \mathrm{O}\right),
$$

where $E(\mathrm{MgO})$ is the energy of the clean $\mathrm{MgO}$ surface (with or without defects), $E\left(\mathrm{H}_{2} \mathrm{O}\right)$ is the energy of an isolated water molecule, and $E\left(\mathrm{MgO} / \mathrm{H}_{2} \mathrm{O}\right)$ is the energy of either the surfacc plus adsorbed water at the equilibrium surface-water distance or the hydroxylated surface optimized as described above.

An energy level diagram showing the Hartree-Fock energies associated with both of these processes at surface, edge, and corner sites on the $\mathrm{MgO}$ surface is given in Fig. 5. As stated before, the binding of water to the perfect surface is favorable by $4.1-5.6 \mathrm{kcal} / \mathrm{mol}$ depending on the orientation of the water molecule over the surface whereas hydroxylating the perfect surface was unfavorable by $24.5 \mathrm{kcal} / \mathrm{mol}$. This picture changes when water is adsorbed at defect sites on the surface. At the four-coordinated sites on the stepedges, the process of water adsorption is energetically favorable and comparable in magnitude to the hydroxylation of these sites. The binding energy associated with water bound perpendicular to the step-edges [see Fig. $1(\mathrm{~b})$ ] is $6.5 \mathrm{kcal} /$ $\mathrm{mol}$, the binding energy for water bound parallel to the stepedge is $10.5 \mathrm{kcal} / \mathrm{mol}$, and hydroxylation of this site is favorable by $7.3 \mathrm{kcal} / \mathrm{mol}$.

For water adsorbed at three-coordinated sites, we find a strong energetic preference for hydroxylation. We examined

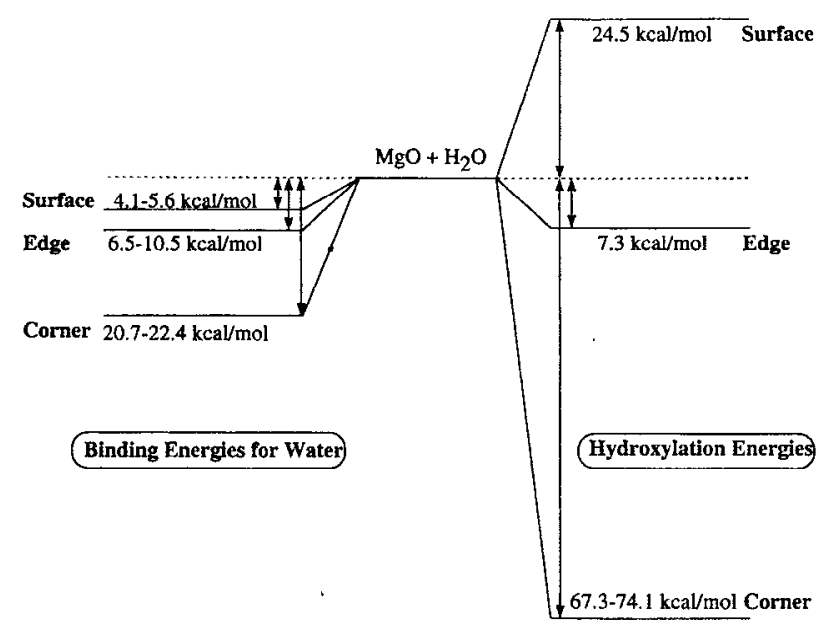

FIG. 5. Hartree-Fock binding and hydroxylation energies for water at various surface defect sites. The surface sites are those described in Ref. 5, the edge sites are those shown in Fig. 1(b), and the corner sites are those of unit cells (b) and (c) in Fig. 3. The dotted line represents the energy of an $\mathrm{MgO}$ surface plus a water molecule at infinite separation.

the binding of water over corner defect configurations defined by unit cells (b) and (c) shown in Fig. 3. The system described by cell (b) where the defect-defect interactions are attractive gives a binding energy of $20.7 \mathrm{kcal} / \mathrm{mol}$ and a hydroxylation energy of $67.3 \mathrm{kcal} / \mathrm{mol}$. When cell (c) is used and the defect interactions are repulsive in nature, the nondissociative adsorption of water gives a binding energy of $22.4 \mathrm{kcal} / \mathrm{mol}$ while hydroxylation of this site results in a net energy gain of $74.1 \mathrm{kcal} / \mathrm{mol}$. The slightly stronger binding in the case of cell (c) indicates that charge buildup between defect sites increases the reactivity of the corner sites.

One additional configuration was examined in connection with binding at corner sites. In Fig. 1(a) a triangle of neighboring oxygen ions at a distance of $2.97 \AA$ are shown at the corner of the defect. These ions produce a region of increased charge density on the surface. In order to determine the effect this region has on the water chemistry at this site, a surface consisting of unit cell (b) with a two-atom thick defect was examined. The binding energy of water in this case was $17.5 \mathrm{kcal} / \mathrm{mol}$, and the corresponding hydroxylation energy was $49.8 \mathrm{kcal} / \mathrm{mol}$. The three-oxygen "triangle" in the one-atom thick defect case enhances both the binding of water and hydroxylation at corner sites. These data indicate that regardless of the defect-defect orientation or step-height, hydroxylation of the corner defect sites is strongly preferred over the nondissociative binding of water. A possible reason for the differences in chemistry at surface, edge, and corner sites can be seen in an analysis of the local charge density about the defect sites.

\section{Mulliken populations}

It is obvious from the reported interaction energies that the reactivity of the low-coordinated defect sites is significantly greater than the fivefold sites on the perfect (001) surface. The change in the character of the oxygen adsorp- 


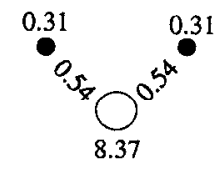

(a)
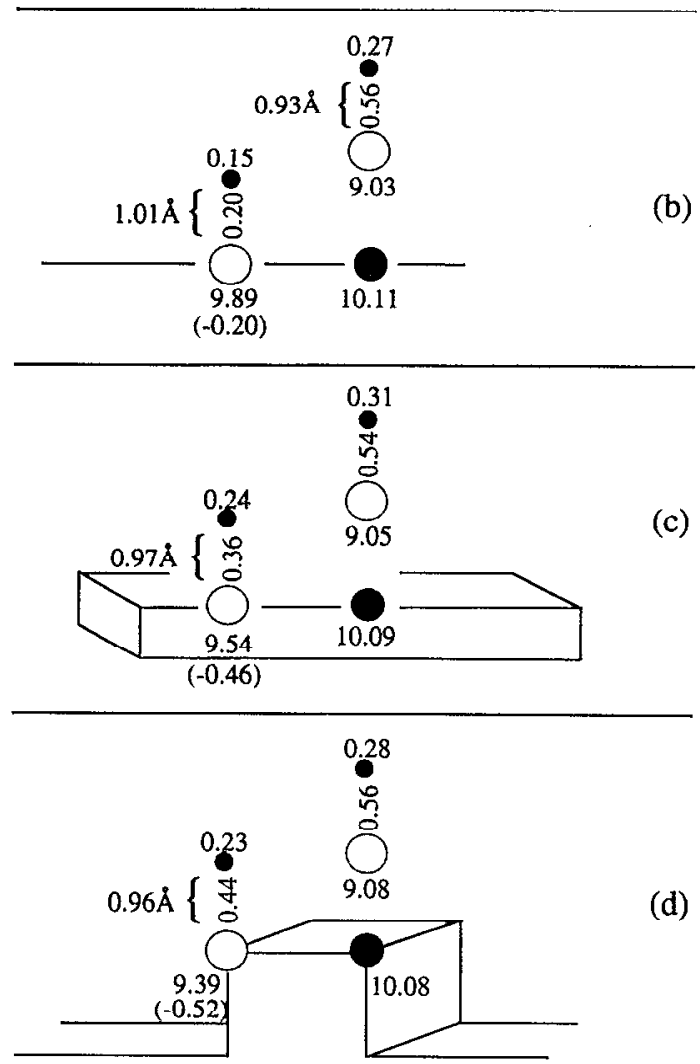

FIG. 6. Mulliken overlap populations for hydroxylated surfaces. (a) Free water, (b) five-coordinated surface sites, (c) four-coordinated edge sites, (d) three-coordinated corner sites. The numbers in parentheses indicate the change in Mulliken population upon the addition of a hydrogen to this site.

tion sites as a function of coordination number can be seen by examining the Mulliken populations of the hydroxylated defect sites and comparing to those found for the hydroxylated perfect (001) surface. Figure 6 shows the Mulliken populations for each of the geometries studied and for a free water molecule, for comparison.

First we will examine the five-, four-, and threecoordinated magnesium site with an $\mathrm{OH}$ group bound to it. In each of these cases, the $\mathrm{Mg}-\mathrm{O}$ and $\mathrm{O}-\mathrm{H}$ distances for hydroxyl groups directly over the surface magnesium ions remain 1.76 and $0.93 \AA$, respectively; these results are consistent with the bond lengths found for an $\mathrm{MgO}$ molecule in the gas phase ${ }^{16}$ and those found for typical hydroxyl containing compounds. The population of electrons on the $\mathrm{Mg}$ adsorption site is constant at $10.11-10.08|e|$ regardless of the coordination number. The atomic and overlap populations of the hydroxyl groups over $\mathrm{Mg}$ also remain the same for the three structures with $0.27-0.31$ electrons on hydrogen, 9.039.08 electrons on oxygen, and an overlap of $0.54-0.56$ electrons between hydrogen and oxygen. Therefore, the differences in the energetics of the hydroxylated surfaces are not substantially affected by the character of the magnesium site.

Next we examine the bond between the remaining hydrogen and the surface oxygen. A theoretical study performed on the dissociation of $\mathrm{H}_{2}$ at structural defects on the $\mathrm{MgO}$ surface concluded that the bond formed between hydrogen and a surface oxygen becomes stronger as the coordination number of the oxygen binding site decreases. ${ }^{17}$ The current study shows a similar trend, and it is the character of this bond that determines the chemistry on these surfaces. Examination of this site in Fig. 6 shows that the character of this bond changes significantly as the local environment around the surface oxygen ion changes. In the most energetically unfavorable case where the hydrogen ion is above a five-coordinated oxygen ion, the $\mathrm{O}-\mathrm{H}$ bond length is $1.10 \AA$, which is significantly longer than the other hydroxyl group. The overlap population associated with this bond is only 0.20 electrons indicating a weak interaction. Consistent with the weak $\mathrm{OH}$ bond, the change in electron population on the surface oxygen site between the clean surface and the hydroxylated surface is small, -0.20 electrons, indicating that the oxygen ion is only weakly perturbed by the presence of the hydrogen. When the hydrogen is bound to an edge site, the equilibrium O-H bond length shortens to $0.97 \AA$, and the population of the $\mathrm{OH}$ bond increases to 0.36 electrons indicating the formation of a stronger bond. Note also that the anlount of charge transferred from the oxygen on the edge site is 0.46 electrons. This is twice that for the case of the five-coordinated oxygen ion. The trend of shorter, stronger bonds continues for the binding of hydroxyl groups to corner sites where the $\mathrm{O}-\mathrm{H}$ overlap population is 0.44 electrons, which approaches the value found in an isolated water molecule. The system containing three-coordinated corner sites greatly favors the formation of stable hydroxyl groups.

\section{Densities of states}

The Mulliken population analysis discussed in the previous section indicated that the oxygen defect sites are fundamentally different from oxygen ions found in the bulk material or the clean surface. This is also apparent in the valence band densities of states resulting from the oxygen ions in the various coordination states. In Fig. 7, the densities of states projected onto the oxygen $2 s$ and $2 p$ orbitals for three-, four-, five-, and six-coordinated oxygen ions on the clean surface are plotted. The bands appearing between -35 and $-25 \mathrm{eV}$ arise from the oxygen $2 s$ orbitals and those in the region -15 to $-5 \mathrm{eV}$ correspond to the oxygen $2 p$ orbitals. The oxygen bands for both five- and six-coordinated ions show similar characteristics. Broad oxygen $2 p$ bands, in each case, fall between -15 and $-9 \mathrm{eV}$. The oxygen $2 s$ bands appear just below $-30 \mathrm{eV}$. The latter is somewhat sharper for the five-coordinated ions than for those in the bulk material. This indicates that this band is somewhat influenced by the bonding within the crystal. When the oxygen ions are fourcoordinated, both oxygen $2 s$ and oxygen $2 p$ bands are much sharper and shifted to higher energies by $1-2 \mathrm{eV}$ from their values in the bulk. The shifts in this case are again small enough that they would be difficult to distinguish in an experimental spectrum. With the introduction of threecoordinated sites, the density of states changes considerably. 


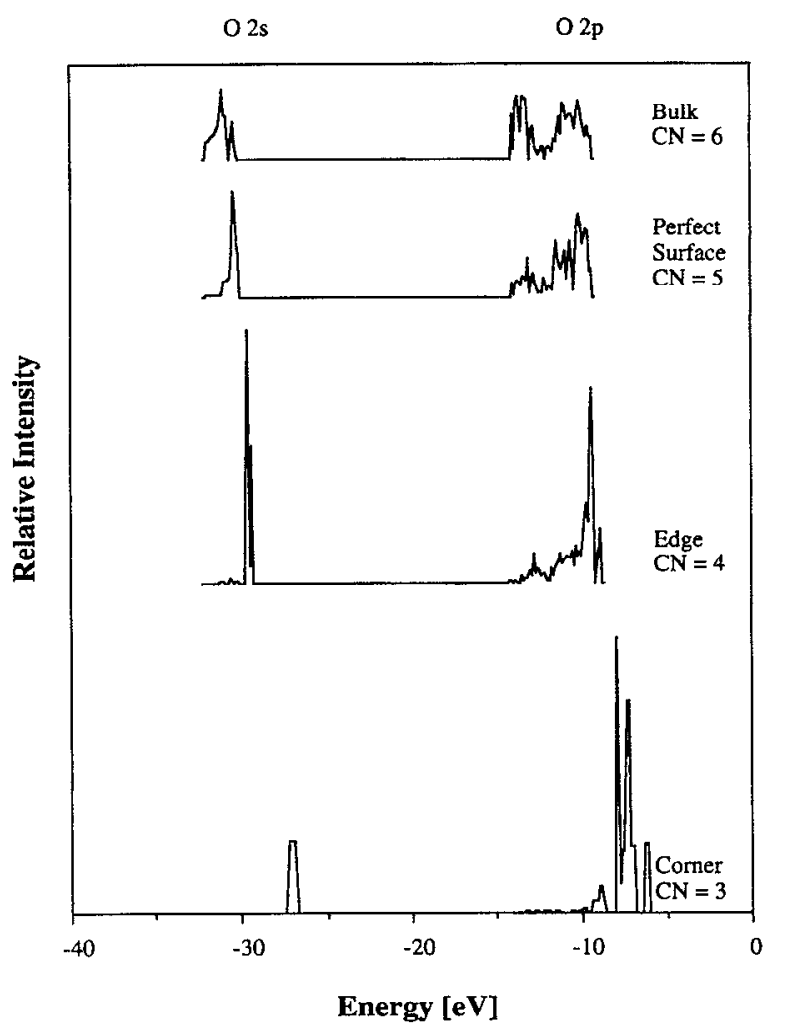

FIG. 7. Densities of states for oxygen at surface defect sites as a function of coordination number. $\mathrm{CN}=6$ are bulk oxygens, 5 surface oxygens, 4 edge oxygens, 3 comer oxygens.

The oxygen $2 s$ band for the three-coordinated site is narrow and higher in energy by almost $5 \mathrm{eV}$ than the oxygen $2 s$ bands for five- and six-coordinated ions. The oxygen $2 p$ band also moves out into the band gap by $3-4 \mathrm{eV}$ indicating that it may be possible to detect these states experimentally using UPS or XPS techniques.

The appearance of surface states due to corner sites correlates with the migration of charge away from the lowcoordinated sites into the bulk material. As the population of electrons in the valence orbitals decreases, their energy rises. It is noteworthy that in the case of the surface containing the three-coordinated defect sites, not only are the oxygen $2 s$ and $2 p$ bands shifted for ions on the defect sites but additional bands also occur in the density of states for the fivecoordinated surface oxygens. These new bands appear at higher energies than those found for the perfect surface. It is evident in Fig. 8 that they arise from surface oxygens directly adjacent to the defect sites.

The shifts in oxygen band energies, along with the decrease in electron population at these sites suggest that the nature of the defect oxygens is different from those within the solid. When an oxygen ion is surrounded by the Madelung field of the crystal, it is stabilized in a highly ionic state $(-1.98$ in bulk $\mathrm{MgO})$. Gas phase $\mathrm{O}^{2-}$ is known to be unstable; hence, it is not surprising that as the coordination number of the oxygen decreases the oxygen ions at these sites become less ionic. The reduced coordination at the sur-

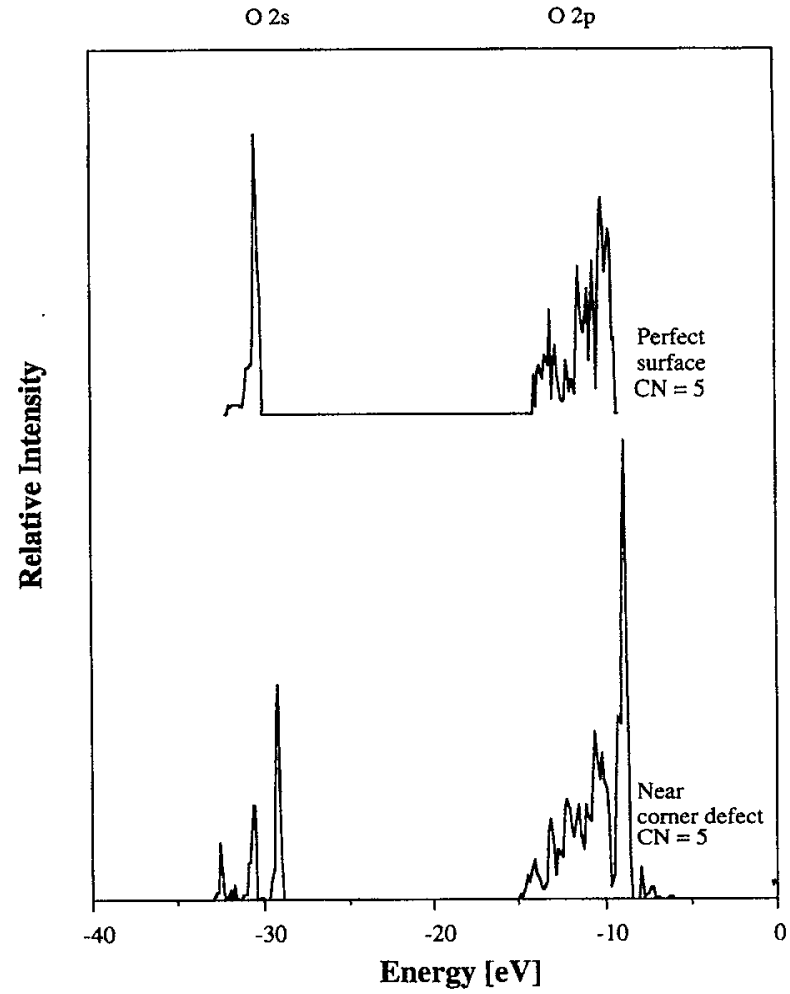

FIG. 8. Densities of states for surface (five-coordinated) oxygen sites near corner defects compared to oxygen ions on a perfect surface.

face sites then facilitates the formation of covalent bonds with the hydrogens resulting from the chemidissociation of water.

\section{E. Hydroxylation of a stretched MgO lattice}

The results presented in the previous sections support the conclusions of the IR, UPS, and XPS studies that hydroxylation of a perfect $\mathrm{MgO}(001)$ surface does not occur. However, we have not yet explained the surface hydroxylation found by HREELS techniques. In the HREELS experiments, the $\mathrm{MgO}$ surface is prepared by depositing magnesium on the (100) surface of a molybdenum substrate such that every Mo atom on the surface is covered by a $\mathrm{Mg}$ atom. ${ }^{18}$ This surface is then exposed to oxygen until it is fully converted to $\mathrm{MgO}$ with the appropriate stoichiometry. It is possible that the thin film created by in this manner has structural entities that promote hydroxylation. There is also a possibility that a slight lattice dilation of the $\mathrm{MgO}$ film caused by a mismatch between the lattice constants of the Mo substrate and bulk $\mathrm{MgO}$ may induce hydroxylation. It is this phenomenon that we address in the following paragraph.

The lattice constant for molybdenum is such that the first layer of the prepared $\mathrm{MgO}$ thin film is stretched by $5.4 \%$ from its equilibrium structure. In order to determine the effect that this perturbation of the surface has on its chemistry, a series of energy points were obtained for the hydroxylated, perfect MgO (001) surface where the lattice constant was varied from 4.0 to $5.0 \AA$. This range of lattice constants en- 


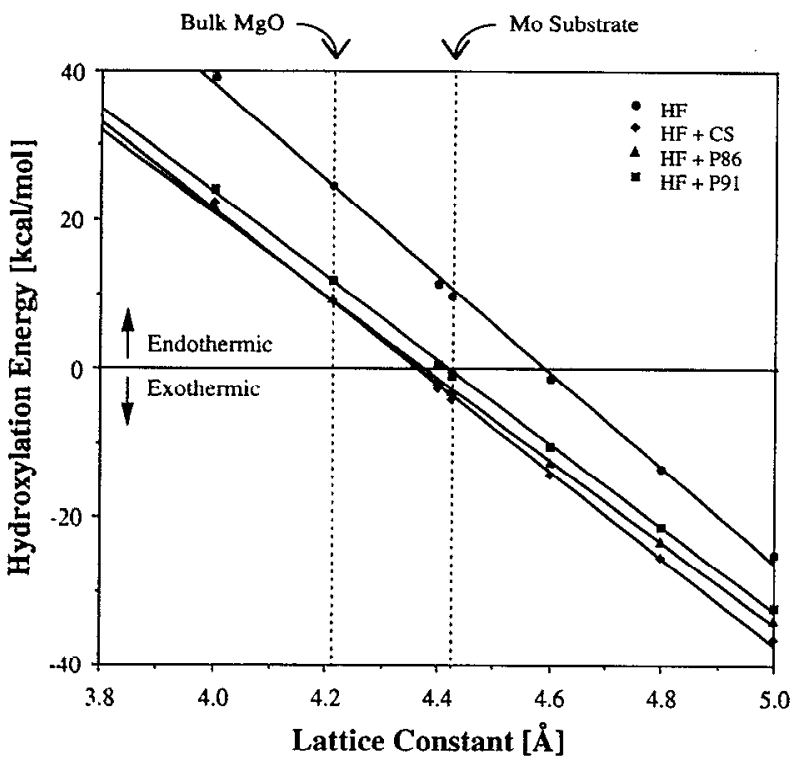

FIG. 9. Hydroxylation energies as a function of MgO lattice constant. Positive energies indicate that hydroxylation is energetically unfavorable and negative energies indicate that hydroxylation is favorable. Dotted lines represent the lattice constants for bulk $\mathrm{MgO}$ and for Mo which is used as a substrate in thin-film $\mathrm{MgO}$ studies. Points labeled HF are the Hartree-Fock energies and those labeled $\mathrm{HF}+\mathrm{CS}, \mathrm{HF}+\mathrm{P} 86$, and $\mathrm{HF}+\mathrm{P} 91$ include Colle Salvetti and Perdew correlation corrections.

compasses those found for bulk $\mathrm{MgO}(4.2 \AA)$ and for the thin film $(4.45 \AA)$. Hydroxylation energies were calculated for these points by referencing each hydroxylated surface to a free water molecule and a clean surface with the corresponding lattice constant. Figure 9 shows these energies to be a linear function of the lattice constant. Hydroxylation of these surfaces is most endothermic at small MgO lattice constants, but at approximately $4.4 \AA$ the correlation corrected hydroxylation energies indicate that this process has become exothermic. The Mulliken populations for the hydroxyl groups on this surface are consistent with our previous observations on hydroxylation at defect sites. The number of electrons populating the $\mathrm{OH}$ group positioned over a surface $\mathrm{Mg}$ ion are identical with those found on the perfect surface with the bulk $\mathrm{MgO}$ lattice constant. The overlap population between the hydrogen and a surface oxygen is $0.26|e|$, which is between that found for the perfect surface and for the edge sites [see Figs. 6(a) and 6(b)]. The populations of $\mathrm{H}$ $(0.15|e|)$ and $O(9.70|e|)$ in this group are also consistent with an $\mathrm{OH}$ bond somewhat stronger than that found on a perfect surface yet weaker than that found on edge sites. These results indicate that hydroxylation of the thin-film $\mathrm{MgO}$ in the HREELS studies may be due to small dilations in the lattice constant. Therefore, the chemistry of oxide surfaces is very sensitive to the method used to prepare the surface.

\section{CONCLUSIONS}

The $a b$ initio calculations performed on water adsorbed on (001) $\mathrm{MgO}$ show that the chemistry of water on this surface is highly dependent on the type of defects present on the surface. Previous work (P1) had shown that the dissociation of water molecules on a defect-free surface was not energetically favorable. The current set of calculations of water on step-edges and corners shows that hydroxylation and molecular adsorption are energetically comparable processes on step-edges and hydroxylation is the energetically dominant process on three-coordinated corner sites.

Mulliken populations show that the surface oxygen sites become less ionic as the coordination number decreases, while the magnesium sites remain relatively unaffected by changes in their local environment. Decreasing the number of oxygen nearest neighbors causes a migration of small amounts of charge from the surface oxygens into the bulk material, allowing oxygen ions at the defect sites to bind more readily to the dissociated hydrogens. The oxygen densities of states corresponding to the valence bands confirm this picture; the oxygen $2 s$ and $2 p$ bands are shifted to higher energies as the coordination number of the oxygen at the defect site decreases. This is another indication that charge is moving away from these sites freeing the oxygen to bind to an incoming hydrogen ion. The process of surface hydroxylation is also sensitive to the lattice constant of the $\mathrm{MgO}$ surface with hydroxylated surfaces becoming more stable as the lattice constant increases. Therefore, the differences in the reported experimental data may be due to distortions in the surface structure caused by different sample preparation methods.

\section{ACKNOWLEDGMENTS}

The authors were supported by the Division of Chemical Sciences Office of Basic Energy Sciences, U. S. Department of Energy under Contract No. DE-AC06-76RLO 1830 with Pacific Northwest Laboratory (M.I.M.) and by the Association of Western Universities (Washington State University) (C.A.S). We also wish to thank the Scientific Computing Staff, Office of Energy Research, U. S. Department of Energy for a grant of computing time at the National Energy Research Supercomputing Center. One of us (N.M.H.) wishes to acknowledge the hospitality of Pacific Northwest Laboratory where this work was performed. Pacific Northwest Laboratory is operated for the U. S. Department of Energy by Battelle Memorial Institute under Contract No. DEAC06-76RLO 1830.

${ }^{1}$ C. F. Jones, R. A. Reeve, R. Rigg, R. L. Segall, R. S. C. Smart, and P. S. Turner, J. Chem. Soc. Faraday Trans. 1 80, 2609 (1984).

${ }^{2}$ H. Onishi, C. Egawa, T. Aruga, and Y. Iwasawa, Surf. Sci. 191, 479 (1987)

${ }^{3}$ M.-C. Wu, C. A. Estrada, J. S. Corneille, and D. W. Goodman, J. Chem. Phys. 96, 3892 (1992).

${ }^{4}$ S. Coluccia, S. Lavagnino, and L. Marchese, Mater. Chem. Phys. 18, 445 (1988).

${ }^{5}$ C. A. Scamehorn, A. C. Hess, and M. I. McCarthy, J. Chem. Phys. 99, 2786 (1993)

${ }^{6}$ R. Dovesi, V. R. Saunders, and C. Roetti, CRYSTAL92 User Manual, Università di Torino, Torino (1992).

${ }^{7}$ M. Causa, R. Colle, A. Fortunelli, R. Dovesi, and C. Pisani, Phys. Scr. 38, 194 (1988).

${ }^{8}$ M. Causa, R. Dovesi, C. Pisani, R. Colle, and A. Fortunelli, Phys. Rev. B 36, 891 (1987).

${ }^{9}$ M. I. McCarthy, A. C. Hess, N. M. Harrison, and V. R. Saunders, J. Chem. Phys. 98, 6387 (1993). 
${ }^{10}$ M. Causa, R. Dovesi, C. Pisani, and C. Roetti, Phys. Rev. B 33, 1308 (1986).

${ }^{11}$ M. Causa, R. Dovesi, E. Kotomin, and C. Pisani, J. Phys. C 20, 4983 (1987).

${ }^{12}$ M. I. McCarthy and A. C. Hess, J. Chem. Phys. 96, 6010 (1992).

${ }^{13}$ W. J. Hehre, L. Radom, P. v. R. Schleyer, and J. A. Pople, Ab Initio Molecular Orbital Theory (Wiley, New York, 1986).

${ }^{14}$ M. J. Frisch, M. Head-Gordon, G. W. Trucks, J. B. Foresman, H. B. Schlegel, K. Raghavachari, M. A. Robb, J. S. Binkley, C. Gonzalez, D. J. Defrees, D. J. Fox, R. A. Whiteside, R. Seeger, C. F. Melius, J. Baker, R.
L. Martin, L. R. Kahn, J. J. P. Stewart, S. Topiol, and J. A. Pople, GAussIAN90 (Gaussian, Inc., Pittsburgh, PA, 1990).

${ }^{15}$ S. S. Kim, S. Baik, H. W. Kim, and C. Y. Kim, Surf. Sci. 294, L935 (1993).

${ }^{16}$ CRC Handbook of Chemistry and Physics (Chemical Rubber, Boca Raton, FL, 1992), Vol. 73, p. 9-19.

${ }^{17}$ E. A. Colbourn, J. Kendrick, and W. C. Mackrodt, Surf. Sci. 126, 550 (1983).

${ }^{18}$ M.-C. Wu, J. S. Corneille, C. A. Estrada, J.-W. He, and D. W. Goodman, Chem. Phys. Lett. 182, 472 (1991). 